\title{
Beyond Genome-Wide Association Studies: New Strategies for Identifying Genetic Determinants of Hypertension
}

\author{
Xiaoling Wang • Bram P. Prins • Siim Sõber • \\ Maris Laan • Harold Snieder \\ Published online: 28 September 2011 \\ (C) The Author(s) 2011. This article is published with open access at Springerlink.com
}

\begin{abstract}
Genetic linkage and association methods have long been the most important tools for gene identification in humans. These approaches can either be hypothesis-based (i.e., candidate-gene studies) or hypothesis-free (i.e., genome-wide studies). The first part of this review offers an overview of the latest successes in gene finding for blood pressure (BP) and essential hypertension using these DNA sequence-based discovery techniques. We further emphasize the importance of post-genome-wide association study (post-GWAS) analysis, which aims to prioritize genetic variants for functional follow-up. Whole-genome next-generation sequencing will eventually be necessary to provide a more comprehensive picture of all DNA variants
\end{abstract}

\author{
B. P. Prins $\cdot$ H. Snieder $(\bowtie)$ \\ Unit of Genetic Epidemiology \& Bioinformatics, \\ Department of Epidemiology, University Medical Center \\ Groningen, University of Groningen, \\ Hanzeplein 1, PO Box 30.001, \\ 9700 RB Groningen, The Netherlands \\ e-mail: h.snieder@umcg.nl \\ B. P. Prins \\ b.p.prins@umcg.nl \\ X. Wang \\ Georgia Prevention Institute, Department of Pediatrics, \\ Medical College of Georgia, \\ Augusta, GA, USA \\ e-mail: xwang@georgiahealth.edu \\ S. Sõber · M. Laan \\ Human Molecular Genetics group, Institute of Molecular and Cell \\ Biology, University of Tartu, \\ Tartu, Estonia \\ S. Sõber \\ e-mail: siims@ut.ee \\ M. Laan \\ e-mail: maris.laan@ut.ee
}

affecting BP and hypertension. The second part of this review discusses promising novel approaches that move beyond the DNA sequence and aim to discover BP genes that are differentially regulated by epigenetic mechanisms, including microRNAs, histone modification, and methylation.

Keywords Linkage analysis - Association analysis . Genome-wide association study · GWAS · Post-GWAS analysis $\cdot$ Next-generation sequencing $\cdot$ Epigenetics . microRNAs $\cdot$ Histone modification $\cdot$ Methylation . Hypertension - Blood pressure

\section{Introduction}

Genetic linkage and association methods have long provided the mainstay of gene-finding efforts in humans, which can either be hypothesis-based (i.e., candidate gene studies) or hypothesis-free (i.e., genome-wide studies) (Table 1). Linkage methods investigate the co-segregation of traits or diseases with genetic markers within family members (e.g., pedigrees or siblings), which proved to be very successful for monogenic (or Mendelian) diseases. For complex traits and diseases such as blood pressure (BP) and essential hypertension, positional cloning (i.e., fine mapping of linkage peaks often harboring a large number of genes) proved unsuccessful, however, because the inheritance of these traits and diseases does not fit a simple Mendelian pattern. In a landmark paper, Risch and Merikangas [1] showed that linkage analysis has minimal power to detect genes for complex traits and diseases. As a consequence, gene-finding efforts have increasingly come to rely on association approaches [2]. Association studies test whether a particular genetic marker and a trait co-occur more often than expected. In contrast to linkage analysis, these studies typically are 
Table 1 DNA sequence-based strategies of gene identification applied to blood pressure (BP) and hypertension (HTN)

\begin{tabular}{|c|c|c|c|}
\hline Strategy & Genetic markers/variants & Mapping resolution $^{\mathrm{a}}$ & Gene identification for BP/HTN? \\
\hline \multicolumn{4}{|l|}{ Hypothesis-based } \\
\hline Candidate gene linkage & Microsatellites & Low & Inconclusive \\
\hline \multicolumn{4}{|l|}{ Candidate gene association ${ }^{\mathrm{b}}$} \\
\hline Direct & Functional SNPs & Moderate & Few replicated \\
\hline Indirect/gene-wide & Set of tagging SNPs & High & Some recent successes \\
\hline Sequenced & All variants & Very high & Some recent successes \\
\hline \multicolumn{4}{|l|}{ Hypothesis-free } \\
\hline Genome-wide linkage & Microsatellites & Low & Monogenic HTN \\
\hline \multicolumn{4}{|l|}{ Genome-wide association } \\
\hline SNP array & SNPs & High & $\mathrm{BP} / \mathrm{HTN}$ in general population \\
\hline Exome sequenced & Coding variants & Very high & Promising \\
\hline Whole genome sequenced & All variants & Very high & Promising \\
\hline
\end{tabular}

${ }^{a}$ The size and accuracy of the mapped genomic region varies as a function of the gene identification method applied. For example, linkage peaks demarcate only broad genomic regions, requiring major fine mapping efforts to identify the underlying causal variants

${ }^{\mathrm{b}}$ Direct candidate-gene association studies typically target a limited number of potentially functional SNPs, whereas indirect (or gene-wide) association studies aim to capture all common variation within the gene using a set of tagging SNPs. Candidate-gene sequencing is often applied to detect and investigate effects of previously unknown rare variants

SNP single nucleotide polymorphism

conducted in unrelated individuals from the general population (but see the review by Ott et al. [3] for the exception to this rule).

Association studies have been greatly facilitated by the sequencing of the human genome, in combination with major efforts to discover single nucleotide polymorphisms (SNPs), such as the International HapMap Project [4]. The increased availability of SNPs resulted in the development of two new association approaches: (1) indirect (or genewide) candidate gene studies considering all common variants within the gene jointly, and (2) the genome-wide association study (GWAS). Rapid improvements in SNP genotyping technology and reductions in cost have now made it feasible to conduct GWASs, which have led to an explosion in the number of newly identified genes for complex traits and diseases [5].

Although developed only recently, large-scale SNP genotyping in the context of GWASs will soon be superseded by next-generation high-throughput DNA sequencing. This technology allows for rapid and efficient sequencing of candidate genes; it is increasingly being applied to whole exomes and even whole genomes [6].

This review first gives an overview of the latest successes in gene finding for BP and hypertension using discovery techniques based on the DNA sequence (Table 1). Results of recent GWASs are not discussed in depth, as they have been reviewed elsewhere [7-9]; instead, we focus on post-GWAS analysis, which aims to prioritize genetic variants for functional follow-up. The second part of this review discusses novel strategies aiming to identify BP genes for which differential regulation of their expression is not coded in their DNA sequence - that is, gene discovery techniques focusing on epigenetic regulation, including microRNAs (miRNAs), histone modification, and methylation.

\section{DNA Sequence-Based Strategies}

\section{Hypothesis-Based (Candidate Gene) Studies}

From 1996 until 2004, over 100 candidate gene linkage studies for BP and hypertension were published, targeting at least 26 candidate genes [10]. Results were largely inconclusive, owing to lack of power and low mapping resolution.

Until recently, candidate gene association studies for BP and hypertension, especially the so-called direct association studies testing only one or two potentially functional SNPs, also yielded few consistently replicated candidates [11]. However, some headway has been made in the past few years in studies using larger sample sizes combined with higher-throughput strategies for genotyping common SNPs and rigorous control of multiple tests.

Newton-Cheh et al. [12 - genotyped a set of 13 SNPs in 14,743 individuals, capturing the majority of common variation at the NPPA-NPPB locus harboring the genes that code for atrial and B-type natriuretic peptides. Strong associations were found for a number of SNPs with both atrial and B-type natriuretic peptides. In 29,717 individuals, the alleles of rs5068 and rs 198358 that showed association 
with increased circulating natriuretic peptide concentrations were also found to be associated with lower systolic BP (SBP) $(0.9-1.5 \mathrm{~mm} \mathrm{Hg})$ and diastolic BP (DBP) (0.3$0.8 \mathrm{~mm} \mathrm{Hg}$ ), as well as reduced odds of hypertension (OR of 0.85 for rs5068 and 0.90 for rs 198358 ).

A number of recent studies employed innovative approaches to investigate association for large numbers of candidate genes. Sõber et al. [13] used a genome-wide genotyping array to test association of common variants with $\mathrm{BP}$ and hypertension in 160 candidate genes. The discovery sample consisted of 1,017 individuals, with follow-up of the most significant SNPs in additional replication cohorts. However, none of the signals survived correction for multiple testing. Tomaszewski et al. [14] used a custom-made gene-centric array with over 30,000 common and rare SNPs to genotype 2,020 European individuals from 520 nuclear families with measures of 24-hour ambulatory BP available. A total of 105 candidate genes for BP, with good genetic coverage of common variants, were present on the array. Nevertheless, little evidence was found for involvement of these most frequently investigated candidate genes for BP, such as those for the sympathetic nervous system and the reninangiotensin system. Interestingly, these results are in line with

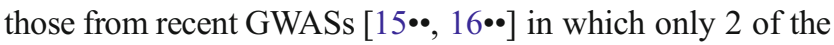
13 gene loci discovered could have been regarded as candidate gene loci for BP (MTHFR and CYP17A1) [7]. However, a final successful study employing a similar strategy indicates that expected effect sizes of common candidate SNPs are small, making large sample sizes a crucial requirement for the detection of their effects. Johnson et al. [17•] investigated 30 gene regions encoding known antihypertensive drug targets in meta-GWAS data from 29,136 individuals of the CHARGE consortium. Nominal evidence of association of $A D R B 1, A D R B 2, A G T, C A C N A 1 A$, $C A C N A 1 C$, and $S L C 12 A 3$ polymorphisms with one or more BP traits was observed. Replication of the top SNPs in the GlobalBPgen Consortium $(n=34,433)$ and the Women's Genome Health Study $(n=23,019)$ resulted in genome-wide significant results $\left(P<5 \times 10^{-8}\right)$ for rs1801253 in ADRB1 (Arg389Gly), with the Gly allele associated with a lower mean SBP $(0.57 \mathrm{~mm} \mathrm{Hg})$ and DBP $(0.36 \mathrm{~mm} \mathrm{Hg})$ and with a SNP in $A G T$ (rs2004776) showing per allele effect sizes of $0.42 \mathrm{~mm} \mathrm{Hg}$ for SBP and $0.32 \mathrm{~mm} \mathrm{Hg}$ for DBP.

In addition to common variants, BP and hypertension may be influenced by rare variants (i.e., allele frequencies $<$ $5 \%$ ) with potentially larger effect sizes. For example, Tomaszewski et al. [14] found a significant overrepresentation of rare variants amongst polymorphisms showing at least nominal association with mean 24-hour BP. These results did not depend on the threshold of rare variant definition (minor allele frequency $[\mathrm{MAF}]<5 \%$ or $\mathrm{MAF}<$ $2 \%)$, significance level $(P<0.05$ or $P<0.01)$, or the phenotype (24-hour SBP or DBP). Resequencing of candidate genes offers an opportunity to detect such rare variants and investigate their association with BP and hypertension. Rao et al. [18] resequenced a locus critical for catecholamine storage, chromogranin A $(C H G A)$, and discovered a lowfrequency $(3 \%)$ functional variant, Gly364Ser, which had a strong effect on autonomic activity as well as DBP. The latter effect was replicated in an independent sample. Similarly, Ji et al. [19॰] resequenced three renal salt-handling genes (SLC12A3, SLC12A1, and KCNJ1) known to be involved in Mendelian syndromes of salt wasting and low BP, in individuals with the most extreme BPs in the population. They discovered a series of rare, nonsynonymous, loss-offunction variants associated with lower BP, clearly showing that rare variants can produce clinically significant BP reduction in the general population.

\section{Hypothesis-Free (Genome-Wide) Studies}

Genome-wide linkage studies have been proven successful in identifying genes underlying monogenic forms of hypertension and hypotension [20,21]. Eighteen such genes have been uncovered so far, with mutations all directly affecting renal tubular electrolyte transport, confirming the key role of the kidney in BP control. Interestingly, there are still some forms of Mendelian hypertension for which the culprit genes remain to be identified [22].

Finding genes for BP and hypertension through GWAS turned out to be even more difficult than for most other complex traits and diseases [7-9]. It was not until a large number of studies joined forces into the GlobalBPGen and CHARGE consortia that 13 novel BP loci were identified $[15 \bullet, 16 \bullet \cdot$. A number of subsequent GWASs have successfully built upon these landmark studies and identified additional loci. After meta-analysis combining the Women's Genome Health Study with prior study results of CHARGE, one suggestive SNP (CASZ1, also replicated in a Japanese study [23]) and one gene expression-associated SNP (BLK-GATA4 region) now reached genome-wide significance [24]. The first successful GWAS for hypertension used an extreme case-control design in a discovery sample of 1,621 hypertension cases and 1,699 hypercontrols, representing the top $2 \%$ and bottom $20 \%$ of the BP distribution in Sweden [25•]. Combined with follow-up validation analyses in 19,845 cases and 16,541 controls, a locus near the Uromodulin (UMOD) gene was identified. $U M O D$ is exclusively expressed in the kidney, suggesting that the discovered variant may have an effect on sodium homeostasis.

Extension of the GWAS approach to other ethnic groups once more emphasizes that large sample sizes, often achieved through meta-GWAS consortia, are essential. For 
example, two initial GWASs in African Americans did not identify any genome-wide significant signals after the replication stages [26, 27]. Admixture mapping and subsequent follow-up association analysis may be a more powerful alternative in such admixed populations, as shown by $\mathrm{Zhu}$ et al. [28•]. They identified a novel variant (rs7726475) on chromosome 5 between the SUB1 and NPR3 genes, associated with both SBP and DBP. Kato et al. [29••] recently published the first large-scale GWAS meta-analysis for SBP and DBP in east Asians, with discovery in 19,608 subjects from eight cohorts and further replication in over 30,000 subjects. They confirmed seven loci previously identified in populations of European descent, but, much more importantly, also identified six novel loci: ST7L-CAPZA1, FIGN-GRB14, ENPEP, NPR3, a newly discovered variant near $T B X 3$, and one near $A L D H 2$. The last locus is likely related to a nonsynonymous SNP (rs167) in $A L D H 2$ that is specific to east Asians. Interestingly, its strong association with both SBP and DBP was largely mediated by alcohol intake.

\section{Post-GWAS Analysis}

An important limitation of GWASs is that genome-wide significant SNPs often merely tag but do not provide direct information on the causal variants. To translate those signals to biological function, follow-up studies are necessary. Recently, the term post-GWAS analysis was coined to refer to this process. The first step of post-GWAS analysis includes a wide variety of mostly bioinformaticsbased (in silico) approaches, aiding prioritization of genes and variants within identified loci for subsequent functional (in vitro and in vivo) follow-up studies (Fig. 1) [30••]. First a window surrounding the top SNP is defined, which is likely to harbor the causal variant or variants. This window is often based on the linkage disequilibrium (LD) structure in the region, because the top SNP is assumed to pick up the association signal through its LD with causal variants. Alternatively, a pragmatically chosen fixed distance can be used, such as $1 \mathrm{Mb}$ on either side of the sentinel SNP, as LD is unlikely to extend beyond this distance [31]. By cross-comparison of the LD structure between populations (e.g., using data from the HapMap project [4]), the region of interest can be further narrowed.

A first glance at all variations of interest is offered by publicly available sequence databases such as those of the 1000 Genomes Project, which comprises whole-genome sequencing data for a large number of individuals representative of African, Asian, and European populations [32•]. It aims to produce a catalogue of human variation with frequencies as low as $0.5 \%$ to $0.1 \%$ and has already identified more than 20 million variants. This resource greatly facilitates investigation of associated regions for

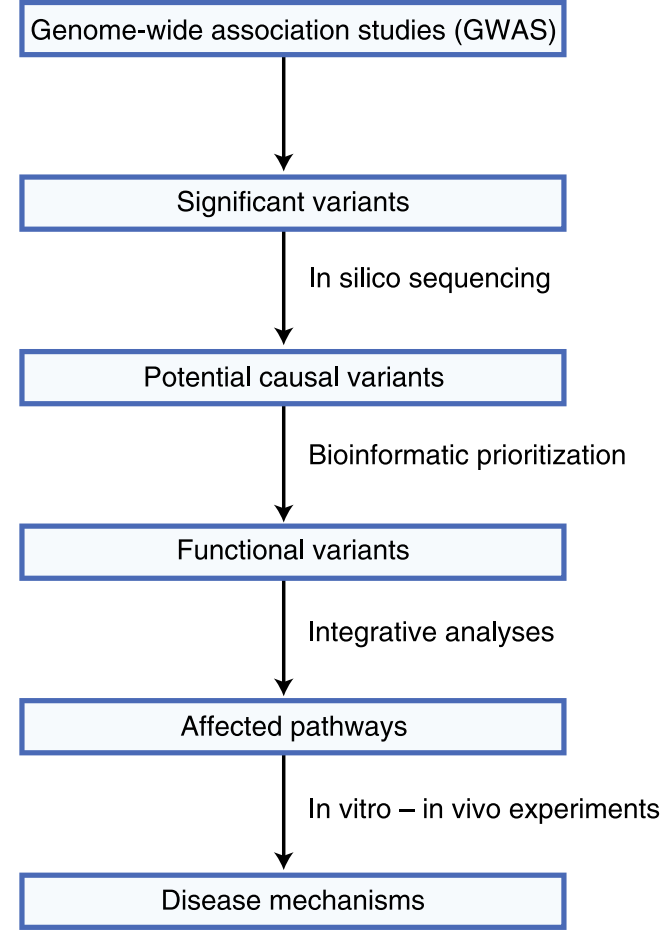

Fig. 1 This diagram illustrates how post-GWAS analysis aids the translation of significant GWAS signals into disease mechanisms

potential causal variants without the need for de novo sequencing. However, if resequencing is deemed necessary, next-generation sequencing is the method of choice. It has significant advantages over traditional methods such as Sanger sequencing or dedicated microarrays in terms of throughput, costs, and coverage of variation [6]. It further allows for detection of copy number variants (CNVs) [33] and variants with lower frequencies, thereby improving the chances of detecting a fuller range of potentially causal polymorphisms.

Once variation within the target region is captured, the genomic properties of the variants can be evaluated using genome browsers such as the one from the University of California Santa Cruz (UCSC) [34] or SNP annotation tools such as WGAViewer [35]. SNPs identified by GWASs often do not map within genes, complicating the interpretation of the findings [36]. To narrow the list of plausible causal variants, bioinformatic approaches are often used that aim to prioritize SNPs based on their potential functionality. These tools apply evaluation criteria such as genomic location, impact on gene expression, likely impact of amino acid substitution on protein function, or previously observed association with other phenotypes. Examples of widely used software allowing such prioritization are PolyPhen [37], SIFT [38], and PupaSuite [39].

Additional information on the functionality of the variants can be derived from integration of GWAS data with "omics" data from transcripts, proteins, and metabo- 
lites, which represent intermediate quantitative molecular traits underlying variation in the phenotypic outcome variables. For example, Annilo et al. [40] demonstrated functional interaction at the RNA level between NPPA and its natural antisense transcript derived from the opposite strand. This might constitute an important posttranscriptional mechanism modulating expression of the NPPA gene, which falls within the MTHFR/NPPA/NPPB locus identified in recent GWASs [16••]. Identified risk variants may affect the causal genes not only at the RNA level (expression quantitative trait loci or eQTLs), but also at the level of the protein (pQTLs) or metabolites (mQTLs). Through integration of GWAS data with these multiple "omics" levels and epigenetic data, this systems genetics approach may provide insight into the causal networks underlying disease etiology [41].

GWAS loci often consist of more than one gene. Prioritization of the most likely causal genes within these loci can be aided by tools that attempt to reconstruct regulatory networks and metabolic pathways in which these genes operate. Depending on the approach, these tools may rely on protein-protein interaction and/or mRNA coexpression databases, as in STRING [42], or on established metabolic networks such as KEGG [43]. Evidence of interaction might also be based on computational prediction, literature mining, or wet-lab experiments.

The above-mentioned methods that aid in translating GWAS findings into actual disease mechanisms depend heavily on computational approaches. Validation of the causality of identified variants and genes requires functional follow-up studies employing both in vitro and in vivo experiments.

\section{Sources of Missing Heritability}

Despite recent successes, the collective effect of BP loci identified through GWASs explains only a small fraction $(<1 \%)$ of BP heritability, which is estimated to range between $30 \%$ and $60 \%$ on the basis of family and twin studies $[44,45]$. Expansion of GWAS sample size in the International Consortium on BP (ICBP)-GWAS, a merger of GlobalBPGen and CHARGE, is likely to uncover additional common BP variants [7], but they will have even smaller effect sizes, and a substantial increase in explained heritability of all loci combined is not expected. Finding suitable answers to the missing heritability enigma is currently the most important challenge in BP and hypertension genetics. Besides additional common variants of small effect, rare variants and structural variations such as copy number variants $(\mathrm{CNVs})$ $(>1 \mathrm{~kb})$ or smaller $(<1 \mathrm{~kb})$ insertion-deletion polymorphisms (indels) are likely sources of missing heritability. Haplotype analysis can be used to extract rare variant effects from the common variants interrogated by the GWAS arrays. Application of this approach to the Wellcome Trust Case Control
Consortium (WTCCC) GWAS data identified rare variants at the ZFAT1 gene affecting hypertension [46]. The WTCCC study also investigated effects of common CNVs on eight complex diseases, including hypertension, but did not detect any associations for hypertension. It is thus unlikely that common $\mathrm{CNVs}$ that can be typed on existing platforms contribute greatly to the missing BP and hypertension heritability [47]. Knowledge of the involvement of indels in complex disease is limited, as no high-throughput detection technology is available. Indels include polymorphic transposable elements, inserted copies of which could interrupt structurally or functionally important genomic regions and affect the expression of a locus. Examples of Alus exhibiting replicated association with BP are the classic polymorphic Alu-insertion (rs4646994: I/D) located in intron 16 of the $A C E$ gene [48] and a recently discovered AluYb8 insertion in intron 10 of the WNK1 gene [49].

Next-generation high-throughput sequencing techniques of whole exomes and genomes (Table 1) are necessary to provide a more comprehensive picture of the variants hidden in the DNA sequence, which may allow us to close at least part of the heritability gap.

\section{Beyond the DNA Sequence: Epigenetics}

Not all gene regulation is coded in the DNA sequence. The second part of this review discusses techniques that aim to discover BP genes that are differentially regulated by epigenetic mechanisms, including miRNAs, histone modification, and methylation.

\section{Role of miRNAs in Blood Pressure Regulation}

miRNAs are small ( $\sim 22$ nucleotides), regulatory, singlestranded RNA molecules, which function by reducing the expression of specific target genes. This method of gene silencing was initially discovered in $C$. elegans, but the mechanism has proven to be prevalent in most multicellular animals [50]. miRNAs are endogenous in origin and are transcribed as longer precursor RNA molecules that are processed into mature miRNAs capable of reducing the translation levels from the targeted mRNA and/or causing its degradation [51]. Currently, more than a thousand miRNAs have been identified in humans [52]. As miRNAs target a large proportion of mammalian genes, they may represent one of the major mechanisms of regulating gene expression, alongside the transcription factors. miRNAs participate in almost all cellular processes, including proliferation, differentiation, and cell death. They modulate developmental processes and cell cycle control and exhibit pervasive dysregulation in cancerous states [53]. There is substantial evidence regarding the roles of microRNAs in 
the development, modeling, and function of various cardiovascular tissues and consequently in cardiovascular disease; extensive reviews of this evidence are available [54-56]. However, the evidence for miRNA involvement in everyday metabolic processes is still limited.

Given the extent of miRNA regulation and the complexity of BP-regulating networks, it is highly likely that miRNAs also modulate BP homeostasis. Although early work on Dahl salt-sensitive rats did not identify differences in miRNA expression profile in the kidneys and heart between animals on a normal diet and a high-salt diet [57], recent studies in genetically similar SS-13BN rats have revealed increased expression of miR-29b in response to high salt intake [58]. Tissue or cell-type specific Dicerknockout mice have demonstrated the importance of miRNAs in the development and maintenance of BPregulating cells. So far, such models have been created for vascular smooth muscle cells (VSMCs) [59•], juxtaglomerular cells [60], and podocytes [61-63]. The loss of miRNAs in juxtaglomerular cells and VSMCs causes significant reductions of BP. In case of juxtaglomerular cells, this difference can be attributed to reduced renin production [60], whereas in VSMCs the reduced BP is due to decreased vascular contractility, which can be attributed to a large extent (but not entirely) to the lack of miR-145 [59•, 64]. In addition to regulating BP by maintaining vascular contractility, several components of the renin-angiotensinaldosterone system have been found to be under miRNA control. One experimentally confirmed example is the mineralocorticoid receptor gene $N R 3 C 2$, downregulated by miRNAs miR-135a and miR-124 [65]. Interestingly, the miRNA-155 binding site in the $3^{\prime}$ untranslated region (UTR) of the angiotensin II receptor 1 (AGTRI) gene carries a polymorphism that leads to differential interaction between mRNA and miRNA and affects BP levels in the general population $[66,67 \bullet \bullet]$. Bioinformatic analyses of the genes in the renin-angiotensin-aldosterone system indicate that several additional genes are potentially regulated by miRNAs $[65$, 68], and the predicted miRNA binding sites contain polymorphisms that may contribute to the interindividual BP variation [68]. For example, a potential polymorphic miRNA binding site has been reported in the $3^{\prime} \mathrm{UTR}$ of the neuropeptide $Y_{1}$ receptor gene [69].

Thus, miRNAs are critically involved in regulating BP, both by maintaining cellular identities in tissues responsible for BP regulation and by maintaining peripheral vascular tension and central regulation of blood volume by modulating the renin-angiotensin-aldosterone system. Improved understanding of the dynamics of the miRNA profile, its effect on the expression of $\mathrm{BP}$ regulating genes, and interplay with genetic variation in the miRNA binding sites may uncover part of the missing BP heritability.
Role of Histone Modification in Blood Pressure Regulation

Histone proteins ( $\mathrm{H} 1-4)$ are major proteins in the chromatin. The four core histones, H2A, H2B, H3, and H4, may undergo a range of posttranslational modifications, including acetylation, methylation, O-GlcNac modification, phosphorylation, SUMOylation, adenosine diphosphate (ADP) ribosylation, and ubiquitination [70]. Histone modifications are indicators of active or repressed chromatin, and the "histone code" hypothesis proposes that combinations of specific histone modifications define chromatin regulation and gene transcription [71, 72]. For example, methylation of histone $\mathrm{H} 3$ on lysines 4 and 36 (H3K4 and H3K36) is generally associated with an open euchromatin structure and transcriptional activation, whereas methylation of histone $\mathrm{H} 3$ on lysines 9 and 27 (H3K9 and H3K27) is generally associated with a closed heterochromatin structure and gene silencing.

Evidence on the role of histone modification in BP regulation derives from animal and in vitro studies on genes causally linked with hypertension. One example is the epithelial sodium channel $(E N a C)$ gene. $E N a C$ plays a major role in sodium reabsorption and hence in the regulation of $\mathrm{BP}$, as evidenced by the findings of mutations in $E N a C$ genes associated with the genetic hypertensive and hypotensive diseases, Liddle's syndrome [73] and pseudohypoaldosteronism type 1 [74]. The studies by Zhang et al. [75] in mouse and cell lines explored whether histone modification can control epithelial sodium channel- $\alpha$ subunit $(E N a C \alpha)$ gene expression in the collecting duct and discovered that a nuclear repressor complex can regulate histone H3 Lys-79 methylation of chromatin associated with the $E N a C \alpha$ promoter and suppress its transcriptional activity. Aldosterone can disrupt this nuclear complex and result in histone H3 Lys79 hypomethylation at specific subregions and derepression of the $E N a C \alpha$ promoter. Another example is WNK lysine-deficient protein kinase 4 (WNK4). The WNK family of serine-threonine kinases has a key role in renal tubular sodium reabsorption. Individuals with pseudohypoaldosteronism II carrying loss-of-function mutations in the WNK4 gene show salt-sensitive hypertension [76]. A recent study by $\mathrm{Mu}$ et al. [77•] in mice on a highsalt diet showed that histone modification plays an important role in decreasing transcription of the WNK4 gene induced by $\beta 2$-adrenergic receptor stimulation. Specifically, isoproterenol-induced transcriptional suppression of the WNK4 gene is mediated by histone acetylation in the promoter region of the $W N K 4$ gene via inhibition of histone deacetylase- 8 activity. These results suggest that histone modification mechanisms affecting these genes are causally linked to hypertension. 
Role of Methylation in Blood Pressure Regulation

DNA methylation, the best known epigenetic modification, involves the addition of a methyl group to cytosines within $\mathrm{CpG}$ pairs. This process is intrinsically linked to the regulation of gene expression. The methylation of these $\mathrm{CpG}$ sites, overrepresented in $\mathrm{CpG}$ islands in the promoter regions of many genes, disrupts the binding of transcription factors and attracts methyl-binding proteins that are associated with gene silencing and chromatin compaction $[78,79]$. Aberrant de novo methylation of $\mathrm{CpG}$ islands is a hallmark of human cancers and is found early during carcinogenesis [80]. Besides cancer, methylation changes of genes have recently been linked to a wide range of complex, often age-related diseases, including hypertension [81], diabetes [82], autoimmune disorders [83], obesity [84], heart disease [85], and mental disorders [86].

In a genome-wide methylation study in Dahl saltsensitive rats, more than 1,000 hypermethylated $\mathrm{CpG}$ sites were identified in the kidneys of salt-sensitive rats compared with normotensive Brown Norway rats. Further pyrosequencing of the promoter of renin genes showed that $10 \mathrm{CpG}$ sites were significantly hypermethylated in saltsensitive rats, consistent with the reduced renin expression in this strain [87]. This study shows evidence that methylation may underlie salt-sensitive hypertension. In addition to salt intake and other postnatal environmental factors, there is now substantial evidence that an adverse fetal environment may program development of hypertension in later life [88-91]. Because DNA methylation takes place in this critical time window, it has been suggested as a good candidate mechanism that may potentially serve as the link between early environmental influences and hypertension [92]. This has been confirmed by Bogdarina et al. [93]. They found significant undermethylation of the proximal promoter of the angiotensin II receptor $A T 1 b$ gene in the adrenals from offspring of mothers fed a low-protein diet. This is consistent with the increased expression of the $A T 1 b$ gene in the adrenal gland and higher BP observed in this rat model. These data suggest that methylation modification of genes represents a link between fetal insults and alteration of gene expression in adult life, leading ultimately to the development of hypertension.

Other genes investigated for epigenetic effects on hypertension in animal studies or in vitro studies are those genes for which exonic mutations have unambiguously been shown to induce hypertension. One example is the 11 beta-hydroxysteroid dehydrogenase type 2 (11 $\beta$-HSD2) gene (HSD11B2). Loss-of-function mutations of this gene lead to a form of salt-sensitive monogenic hypertension [94]. Methylation modulation of this gene has recently been demonstrated in both a rodent model and cultured human cell lines [95]. CpG islands covering the promoter and first exon of HSD11B2 were found to be densely methylated in tissues and cell lines with low expression but not in those with high expression of $11 \beta-H S D 2$. Demethylation enhanced the transcription and activity of the enzyme in human cells in vitro and in rats in vivo. Methylation of HSD11B2 promoterluciferase constructs decreased transcriptional activity, and methylation of recognition sequence of transcription factors known to be relevant for the expression of this enzyme diminished their binding activity [95]. A recent study [96] also showed increased methylation of several $\mathrm{CpG}$ promoter sites of HSD11B2 in kidneys from rat offspring suffering from intrauterine growth restriction.

Data on humans are scarce. In one study, Friso et al. [97] measured promoter methylation of the HSD11B2 gene in peripheral-blood mononuclear cells from patients with hypertension and 32 patients on prednisone therapy. Elevated $H S D 11 B 2$ promoter methylation was associated with decreased HSD11B2 activity and hypertension development in glucocorticoid-treated patients. In a recent study by Smolarek et al. [98], significantly lower global DNA methylation levels were observed in patients with hypertension. Further evidence comes from studies on DNA methylation profiling of preeclampsia, a disease characterized by high $\mathrm{BP}$ in pregnancy. Both candidate-gene and genome-wide approaches have confirmed that epigenetic features are implicated in the pathogenesis of preeclampsia. For example, in a recent genome-wide methylation analysis [99], differentially methylated $\mathrm{CpG}$ sites were identified in a genome scan of cases with early-onset preeclampsia versus controls. Four loci could be further replicated in a larger sample, and one locus was shown to affect gene expression.

Based on the convincing evidence from animal studies and the promising evidence from small-scale human studies and from hypertension-related diseases, large-scale epigenomewide human studies are needed not only to confirm the role of methylation mechanisms in hypertension but also to identify the specific $\mathrm{CpG}$ sites responsible for the global methylation differences in hypertension.

\section{Conclusions}

GWAS has proved to be a breakthrough technology for identification of genetic determinants of BP and hypertension, although so far it has allowed us only to scratch the surface, with most of the heritability still missing [9]. Moreover, additional work is needed to translate genomewide significant signals to biological function, with postGWAS analysis constituting a crucial first step. Finding suitable answers to the missing heritability problem is currently the most important challenge in BP and hyper- 
tension genetics. Whole-genome sequencing may eventually be necessary to provide a more comprehensive picture of all DNA variants affecting BP and hypertension. Recently, some promising approaches have emerged that move beyond the DNA sequence and focus on identification of BP genes regulated by epigenetic mechanisms such as miRNAs, histone modification, and methylation. Such studies are urgently needed, not only to identify epigenetic variants that may contribute to the missing heritability, but perhaps even more importantly, to identify those that mediate differential gene expression resulting from environmental influences.

Disclosure No potential conflicts of interest relevant to this article were reported.

Open Access This article is distributed under the terms of the Creative Commons Attribution Noncommercial License which permits any noncommercial use, distribution, and reproduction in any medium, provided the original author(s) and source are credited.

\section{References}

Papers of particular interest, published recently, have been highlighted as:

- Of importance

- Of major importance

1. Risch N, Merikangas K. The future of genetic studies of complex human diseases. Science. 1996;273(5281):1516-7.

2. Nolte IM, McCaffery JM, Snieder H. Candidate gene and genome-wide association studies in behavioral medicine. In: Steptoe A, editor. Handbook of behavioral medicine: Methods and applications. New York: Springer; 2010. p. 423-41.

3. Ott J, Kamatani Y, Lathrop M. Family-based designs for genomewide association studies. Nat Rev Genet. 2011;12(7):465-74.

4. Frazer KA, Ballinger DG, Cox DR, et al. A second generation human haplotype map of over 3.1 million SNPs. Nature. 2007;449 (7164):851-61.

5. Hindorff LA, Junkins HA, Mehta JP, Manolio TA: A catalog of published Genome-Wide Association Studies. Available at: www. genome.gov/gwastudies Accessed in July 2011.

6. Metzker ML. Sequencing technologies - the next generation. Nat Rev Genet. 2010;11(1):31-46.

7. Ehret GB. Genome-wide association studies: contribution of genomics to understanding blood pressure and essential hypertension. Curr Hypertens Rep. 2010;12(1):17-25.

8. Zhang K, Weder AB, Eskin E, O'Connor DT. Genome-wide case/ control studies in hypertension: only the 'tip of the iceberg'. J Hypertens. 2010;28(6):1115-23.

9. Wang X, Snieder H. Genome-wide association studies and beyond: what's next in blood pressure genetics? Hypertension. 2010;56(6):1035-7.

10. Cowley Jr AW. The genetic dissection of essential hypertension. Nat Rev Genet. 2006;7(11):829-40.

11. Luft FC. Geneticism of essential hypertension. Hypertension. 2004;43(6):1155-9.

12. - Newton-Cheh C, Larson MG, Vasan RS, et al.: Association of common variants in NPPA and NPPB with circulating natriuretic peptides and blood pressure. Nat Genet 2009, 41(3):348-353. This large-scale candidate gene study showed that SNPs in the genes that code for atrial and B-type natriuretic peptides also show association with systolic and diastolic BP.

13. Sõber S, Org E, Kepp K, et al. Targeting 160 candidate genes for blood pressure regulation with a genome-wide genotyping array. PLoS One. 2009;4(6):e6034.

14. Tomaszewski M, Debiec R, Braund PS, et al. Genetic architecture of ambulatory blood pressure in the general population: insights from cardiovascular gene-centric array. Hypertension. 2010;56 (6):1069-76.

15. • Levy D, Ehret GB, Rice K, et al.: Genome-wide association study of blood pressure and hypertension. Nat Genet 2009, 41 (6):677-687. This paper and the one by Newton-Cheh et al. [16••] were the first successful GWAS meta-analyses for blood pressure, identifying 13 independent BP loci.

16. •- Newton-Cheh C, Johnson T, Gateva V, et al.: Genome-wide association study identifies eight loci associated with blood pressure. Nat Genet 2009, 41(6):666-676. This paper and the one by Levy et al. [15•0] were the first successful GWAS metaanalyses for blood pressure, identifying 13 independent BP loci.

17. - Johnson AD, Newton-Cheh C, Chasman DI, et al.: Association of hypertension drug target genes with blood pressure and hypertension in 86,588 individuals. Hypertension 2011, 57 (5):903-910. This study shows that candidate gene studies for $B P$ and hypertension may be successful if using meta-GWAS-type sample sizes.

18. Rao F, Wen G, Gayen JR, et al. Catecholamine release-inhibitory peptide catestatin (chromogranin A(352-372)): naturally occurring amino acid variant Gly364Ser causes profound changes in human autonomic activity and alters risk for hypertension. Circulation. 2007;115(17):2271-81.

19. - Ji W, Foo JN, O'Roak BJ, et al.: Rare independent mutations in renal salt handling genes contribute to blood pressure variation. Nat Genet 2008, 40(5):592-599. This landmark study proved that rare variants may have a considerable effect on $B P$ in the general population.

20. Lifton RP, Gharavi AG, Geller DS. Molecular mechanisms of human hypertension. Cell. 2001;104(4):545-56.

21. Vehaskari VM. Heritable forms of hypertension. Pediatr Nephrol. 2009;24(10):1929-37.

22. Carrs KJ, Stowasser M, Gordon RD, O'Shaughnessy KM. Further study of chromosome $7 \mathrm{p} 22$ to identify the molecular basis of familial hyperaldosteronism type II. J Hum Hypertens. 2011;25 (9):560-4.

23. Takeuchi $\mathrm{F}$, Isono $\mathrm{M}$, Katsuya $\mathrm{T}$, et al. Blood pressure and hypertension are associated with 7 loci in the Japanese population. Circulation. 2010;121(21):2302-9.

24. Ho JE, Levy D, Rose L, et al. Discovery and replication of novel blood pressure genetic loci in the Women's Genome Health Study. J Hypertens. 2010;29(1):62-9.

25. - Padmanabhan S, Melander O, Johnson T, et al.: Genome-wide association study of blood pressure extremes identifies variant near UMOD associated with hypertension. PLoS Genet 2010, 6 (10):e1001177. This first successful GWAS for hypertension used an extreme case-control design and identified a locus near the Uromodulin gene.

26. Adeyemo A, Gerry N, Chen G, et al. A genome-wide association study of hypertension and blood pressure in African Americans. PLoS Genet. 2009;5(7):e1000564.

27. Fox ER, Young JH, Li Y, et al. Association of genetic variation with systolic and diastolic blood pressure among African Americans: the Candidate Gene Association Resource study. Hum Mol Genet. 2011;20(11):2273-84.

28. - Zhu X, Young JH, Fox E, et al.: Combined admixture mapping and association analysis identifies a novel blood pressure genetic locus on 5p13: contributions from the CARe consortium. Hum 
Mol Genet 2011, 20(11):2285-2295. This paper shows that admixture mapping combined with large-scale follow-up association analysis may be a more powerful alternative than GWAS in admixed populations such as African Americans.

29. •- Kato N, Takeuchi F, Tabara Y, et al.: Meta-analysis of genomewide association studies identifies common variants associated with blood pressure variation in east Asians. Nat Genet 2011, 43 (6):531-538. This study shows that the meta-GWAS formula can be successfully transferred to other ethnic groups. In large sample sizes of east Asians, seven European BP loci were confirmed and six novel loci identified.

30. • Freedman ML, Monteiro AN, Gayther SA, et al.: Principles for the post-GWAS functional characterization of cancer risk loci. Nat Genet 2011, 43(6):513-518. This is the first study to highlight the importance of post-GWAS analysis and comprehensively describe its principles.

31. Pritchard JK, Przeworski M. Linkage disequilibrium in humans: models and data. Am J Hum Genet. 2001;69(1):1-14.

32. The 1000 Genomes Project Consortium. A map of human genome variation from population-scale sequencing. Nature 2010, 467(7319):1061-1073. This paper describes the aims of the 1000 Genomes Project and provides the results of its pilot phase.

33. Medvedev P, Stanciu M, Brudno M. Computational methods for discovering structural variation with next-generation sequencing. Nat Methods. 2009;6(11 Suppl):S13-20.

34. Kent WJ, Sugnet CW, Furey TS, et al. The human genome browser at UCSC. Genome Res. 2002;12(6):996-1006.

35. Ge D, Zhang K, Need AC, et al. WGAViewer: software for genomic annotation of whole genome association studies. Genome Res. 2008;18(4):640-3.

36. Hindorff LA, Sethupathy P, Junkins HA, et al. Potential etiologic and functional implications of genome-wide association loci for human diseases and traits. Proc Natl Acad Sci U S A. 2009;106(23):9362-7.

37. Adzhubei IA, Schmidt S, Peshkin L, et al. A method and server for predicting damaging missense mutations. Nat Methods. 2010;7(4):248-9.

38. Ng PC, Henikoff S. SIFT: predicting amino acid changes that affect protein function. Nucleic Acids Res. 2003;31(13):3812-4.

39. Conde L, Vaquerizas JM, Dopazo H, et al.: PupaSuite: finding functional single nucleotide polymorphisms for large-scale genotyping purposes. Nucleic Acids Res 2006, 34(Web Server issue):W621-625.

40. Annilo T, Kepp K, Laan M. Natural antisense transcript of natriuretic peptide precursor A (NPPA): structural organization and modulation of NPPA expression. BMC Mol Biol. 2009;10:81.

41. Quigley D, Balmain A. Systems genetics analysis of cancer susceptibility: from mouse models to humans. Nat Rev Genet. 2009;10(9):651-7.

42. Szklarczyk D, Franceschini A, Kuhn M, et al.: The STRING database in 2011: functional interaction networks of proteins, globally integrated and scored. Nucleic Acids Res 2011, 39 (Database issue):D561-568.

43. Kanehisa M, Goto S, Furumichi M, et al.: KEGG for representation and analysis of molecular networks involving diseases and drugs. Nucleic Acids Res 2010, 38(Database issue):D355-360.

44. Snieder H, Harshfield GA, Dekkers JC, Treiber FA. Heritability of resting hemodynamics in African and European American youth. Hypertension. 2003;41:1196-201.

45. Wang X, Snieder H. Familial aggregation of blood pressure. In: Flynn JT, Ingelfinger JR, Portman RJ, editors. Clinical hypertension and vascular diseases: pediatric hypertension. 2nd ed. Totowa: Humana Press Inc; 2011. p. 241-58.

46. Feng T, Zhu X. Genome-wide searching of rare genetic variants in WTCCC data. Hum Genet. 2010;128(3):269-80.

47. Craddock N, Hurles ME, Cardin N, et al. Genome-wide association study of CNVs in 16,000 cases of eight common diseases and 3,000 shared controls. Nature. 2010;464(7289):713-20.
48. Rigat B, Hubert C, Alhenc-Gelas F, et al. An insertion/deletion polymorphism in the angiotensin I-converting enzyme gene accounting for half the variance of serum enzyme levels. J Clin Invest. 1990;86(4):1343-6.

49. Putku M, Kepp K, Org E, et al. Novel polymorphic AluYb8 insertion in the WNK1 gene is associated with blood pressure variation in Europeans. Hum Mutat. 2011;32(7):806-14.

50. Bartel DP. MicroRNAs: target recognition and regulatory functions. Cell. 2009;136(2):215-33.

51. Guo H, Ingolia NT, Weissman JS, Bartel DP. Mammalian microRNAs predominantly act to decrease target mRNA levels. Nature. 2010;466(7308):835-40.

52. Kozomara A, Griffiths-Jones S: miRBase: integrating microRNA annotation and deep-sequencing data. Nucleic Acids Res 2011, 39 (Database issue):D152-157.

53. Volinia S, Galasso M, Costinean S, et al. Reprogramming of miRNA networks in cancer and leukemia. Genome Res. 2010;20 (5):589-99.

54. Ohtani K, Dimmeler S. Control of cardiovascular differentiation by microRNAs. Basic Res Cardiol. 2011;106(1):5-11.

55. Zhang C. MicroRNAs in vascular biology and vascular disease. J Cardiovasc Transl Res. 2010;3(3):235-40.

56. Small EM, Olson EN. Pervasive roles of microRNAs in cardiovascular biology. Nature. 2011;469(7330):336-42.

57. Naraba H, Iwai N. Assessment of the microRNA system in saltsensitive hypertension. Hypertens Res. 2005;28(10):819-26.

58. Liu Y, Taylor NE, Lu L, et al. Renal medullary microRNAs in Dahl salt-sensitive rats: miR-29b regulates several collagens and related genes. Hypertension. 2010;55(4):974-82.

59. - Albinsson S, Skoura A, Yu J, et al.: Smooth muscle miRNAs are critical for post-natal regulation of blood pressure and vascular function. PLoS One 2011, 6(4):e18869. This study demonstrates that in addition to embryonic development, miRNAs are also important in maintaining vascular smooth muscle cells and BP homeostasis in adult organisms.

60. Sequeira-Lopez ML, Weatherford ET, Borges GR, et al. The microRNA-processing enzyme dicer maintains juxtaglomerular cells. J Am Soc Nephrol. 2010;21(3):460-7.

61. Harvey SJ, Jarad G, Cunningham J, et al. Podocyte-specific deletion of dicer alters cytoskeletal dynamics and causes glomerular disease. J Am Soc Nephrol. 2008;19(11):2150-8.

62. Ho J, Ng KH, Rosen S, et al. Podocyte-specific loss of functional microRNAs leads to rapid glomerular and tubular injury. J Am Soc Nephrol. 2008;19(11):2069-75.

63. Shi S, Yu L, Chiu C, et al. Podocyte-selective deletion of dicer induces proteinuria and glomerulosclerosis. J Am Soc Nephrol. 2008;19(11):2159-69.

64. Xin M, Small EM, Sutherland LB, et al. MicroRNAs miR-143 and miR-145 modulate cytoskeletal dynamics and responsiveness of smooth muscle cells to injury. Genes Dev. 2009;23(18):2166-78.

65. Sober S, Laan M, Annilo T. MicroRNAs miR-124 and miR-135a are potential regulators of the mineralocorticoid receptor gene (NR3C2) expression. Biochem Biophys Res Commun. 2010;391 (1):727-32.

66. Ceolotto G, Papparella I, Bortoluzzi A, et al. Interplay between miR155, AT1R A1166C polymorphism, and AT1R expression in young untreated hypertensives. Am J Hypertens. 2011;24(2):241-6.

67. •• Sethupathy P, Borel C, Gagnebin M, et al.: Human microRNA155 on chromosome 21 differentially interacts with its polymorphic target in the AGTR1 3' untranslated region: a mechanism for functional single-nucleotide polymorphisms related to phenotypes. Am J Hum Genet 2007, 81(2):405-413. This is the first report of a polymorphism in an miRNA binding site that affects blood pressure.

68. Elton TS, Sansom SE, Martin MM: Cardiovascular Disease, Single Nucleotide Polymorphisms; and the Renin Angiotensin 
System: Is There a MicroRNA Connection? Int J Hypertens 2010, published online 2010 Aug 4. doi:10.4061/2010/281692.

69. Wang L, Rao F, Zhang K, et al. Neuropeptide Y(1) Receptor NPY1R discovery of naturally occurring human genetic variants governing gene expression in cella as well as pleiotropic effects on autonomic activity and blood pressure in vivo. J Am Coll Cardiol. 2009;54(10):944-54.

70. Marmorstein R, Trievel RC. Histone modifying enzymes: structures, mechanisms, and specificities. Biochim Biophys Acta. 2009; 1789(1):58-68.

71. Berger SL. The complex language of chromatin regulation during transcription. Nature. 2007;447(7143):407-12.

72. Jenuwein T, Allis CD. Translating the histone code. Science, 2001;293(5532):1074-80.

73. Tamura H, Schild L, Enomoto N, et al. Liddle disease caused by a missense mutation of beta subunit of the epithelial sodium channel gene. J Clin Invest. 1996;97(7):1780-4.

74. Bonny O, Knoers N, Monnens L, Rossier BC. A novel mutation of the epithelial $\mathrm{Na}+$ channel causes type 1 pseudohypoaldosteronism. Pediatr Nephrol. 2002;17(10):804-8.

75. Zhang D, Yu ZY, Cruz P, et al. Epigenetics and the control of epithelial sodium channel expression in collecting duct. Kidney Int. 2009;75(3):260-7.

76. Wilson FH, Disse-Nicodeme S, Choate KA, et al. Human hypertension caused by mutations in WNK kinases. Science. 2001;293(5532):1107-12.

77. - Mu S, Shimosawa T, Ogura S, et al.: Epigenetic modulation of the renal beta-adrenergic-WNK4 pathway in salt-sensitive hypertension. Nat Med 2011, 17(5):573-580. This study implicates the epigenetic modulation of WNK4 transcription in the development of salt-sensitive hypertension.

78. Holliday R, Pugh JE. DNA modification mechanisms and gene activity during development. Science. 1975;187(4173):226-32.

79. Riggs AD. $X$ inactivation, differentiation, and DNA methylation. Cytogenet Cell Genet. 1975;14(1):9-25.

80. Jones PA, Baylin SB. The epigenomics of cancer. Cell. 2007;128 (4):683-92.

81. Frey FJ. Methylation of $\mathrm{CpG}$ islands: potential relevance for hypertension and kidney diseases. Nephrol Dial Transplant. 2005;20(5):868-9.

82. Muhonen P, Holthofer H. Epigenetic and microRNA-mediated regulation in diabetes. Nephrol Dial Transplant. 2009;24(4):1088-96.

83. Wilson AG. Epigenetic regulation of gene expression in the inflammatory response and relevance to common diseases. J Periodontol. 2008;79(8 Suppl):1514-9.

84. Wang X, Zhu H, Snieder H, et al. Obesity related methylation changes in DNA of peripheral blood leukocytes. BMC Med. 2010;8:87.

85. Lund G, Andersson L, Lauria M, et al. DNA methylation polymorphisms precede any histological sign of atherosclerosis in mice lacking apolipoprotein E. J Biol Chem. 2004;279 (28):29147-54

86. Mill J, Tang T, Kaminsky Z, et al. Epigenomic profiling reveals DNA-methylation changes associated with major psychosis. Am J Hum Genet. 2008;82(3):696-711.

87. Michalkiewicz M, Michalkiewicz T, MacGillis K: Combined epigenomic and genomic approach to hypertension. The FASEB Journal 2008, 22 (Meeting Abstract Suppl):968.

88. Law CM, Shiell AW. Is blood pressure inversely related to birth weight? The strength of evidence from a systematic review of the literature. J Hypertens. 1996;14(8):935-41.

89. Poulter NR, Chang CL, MacGregor AJ, et al. Association between birth weight and adult blood pressure in twins: historical cohort study. BMJ. 1999;319(7221):1330-3.

90. McNeill G, Tuya C, Smith WC. The role of genetic and environmental factors in the association between birthweight and blood pressure: evidence from meta-analysis of twin studies. Int $\mathrm{J}$ Epidemiol. 2004;33(5):995-1001.

91. Bergvall N, Iliadou A, Johansson S, et al. Genetic and shared environmental factors do not confound the association between birth weight and hypertension: a study among Swedish twins. Circulation. 2007;115(23):2931-8.

92. Gallou-Kabani C, Junien C. Nutritional epigenomics of metabolic syndrome: new perspective against the epidemic. Diabetes. 2005;54(7):1899-906.

93. Bogdarina I, Welham S, King PJ, et al. Epigenetic modification of the renin-angiotensin system in the fetal programming of hypertension. Circ Res. 2007;100(4):520-6.

94. Lovati E, Ferrari P, Dick B, et al. Molecular basis of human salt sensitivity: the role of the 11beta-hydroxysteroid dehydrogenase type 2. J Clin Endocrinol Metab. 1999;84(10):3745-9.

95. Alikhani-Koopaei R, Fouladkou F, Frey FJ, Frey BM. Epigenetic regulation of 11 beta-hydroxysteroid dehydrogenase type 2 expression. J Clin Invest. 2004;114(8):1146-57.

96. Baserga M, Kaur R, Hale MA, et al. Fetal growth restriction alters transcription factor binding and epigenetic mechanisms of renal 11beta-hydroxysteroid dehydrogenase type 2 in a sex-specific manner. Am J Physiol Regul Integr Comp Physiol. 2010;299(1): R334-342.

97. Friso S, Pizzolo F, Choi SW, et al. Epigenetic control of 11 betahydroxysteroid dehydrogenase 2 gene promoter is related to human hypertension. Atherosclerosis. 2008;199(2):323-7.

98. Smolarek I, Wyszko E, Barciszewska AM, et al.: Global DNA methylation changes in blood of patients with essential hypertension. Med Sci Monit 2010, 16(3):CR149-155.

99. Yuen RK, Penaherrera MS, von Dadelszen P, et al. DNA methylation profiling of human placentas reveals promoter hypomethylation of multiple genes in early-onset preeclampsia. Eur J Hum Genet. 2010;18(9):1006-12. 